\title{
Evaluación del riesgo quirúrgico en pacientes con cirrosis hepática
}

\section{Surgical risk assessment in patients with liver cirrosis}

Óscar Santos Sánchez, MD.1*

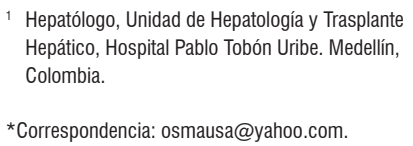

*Correspondencia: osmausa@yahoo.com.

Fecha recibido: $29-01-18$

Fecha aceptado: 06-11-18

\begin{abstract}
Resumen
Los pacientes cirróticos pueden requerir procedimientos quirúrgicos y están relacionados con mayor morbimortalidad que la población general. Los resultados adversos están asociados con múltiples factores, pero los más importantes son la severidad de la enfermedad hepática, la urgencia del procedimiento y el tipo de cirugía. El puntaje Child-Turcott-Pugh (CTP) y el puntaje MELD pueden ser usados para determinar la severidad de la enfermedad hepática y estratificar el riesgo. Se considera que la cirugía electiva se tolera bien en pacientes con CTP A, es permisible con una buena preparación preoperatoria en pacientes con CTP B o MELD $<14$ y está contraindicada en pacientes con CTP C o MELD $>15$ con albúmina $<2,5 \mathrm{~g} / \mathrm{dL}$. Lo ideal son procedimientos electivos, mínimamente invasivos y que sean realizados en centros especializados y con médicos entrenados en el cuidado de este tipo de pacientes. La optimización preoperatoria después de la estratificación del riesgo y un cuidadoso manejo son obligatorios antes y después de la cirugía. Una aproximación multidisciplinaria, individualizada y especializada puede mejorar los resultados.
\end{abstract}

\section{Palabras clave}

Riesgo quirúrgico, cirrosis, cirugía, resultados, Child, MELD.

\begin{abstract}
Cirrhotic patients may require surgical procedures and face higher morbidity and mortality than the general population. Adverse results are associated with multiple factors, but the most important are the severity of the liver disease, the urgency of the procedure and the type of surgery. The Child-Turcott-Pugh score (CTP) and the MELD score can be used to determine the severity of liver disease and to stratify the risk. Patients with CTP A are considered to tolerate elective surgery well, and surgery is permissible in patients with CTP B or MELD $<14$ with good preoperative preparation. It is contraindicated in patients with CTP C or MELD $>15$ with albumin $<2.5 \mathrm{gr} / \mathrm{dL}$. Ideally, elective, minimally invasive procedures are used in specialized centers with doctors trained in the care of this type of patient. Preoperative optimization after risk stratification and careful management are mandatory before and after surgery. A multidisciplinary, individualized and specialized approach can improve results.
\end{abstract}

\section{Keywords}

Surgical risk, cirrhosis, surgery, results, Child, MELD.

\section{INTRODUCCIÓN}

Los procedimientos quirúrgicos en pacientes con enfermedad hepática conllevan un riesgo significativo de complica- ciones que pueden ser graves y pueden generar alta morbimortalidad. La magnitud del riesgo quirúrgico depende de varios factores que incluyen la gravedad de la enfermedad hepática, el tipo y localización del procedimiento quirúr- 
gico, la urgencia del mismo, el tipo de anestesia y las comorbilidades (1). Aunque la resección hepática de tumores y el trasplante hepático son las principales cirugías en pacientes cirróticos, estos pacientes frecuentemente necesitan otro tipo de procedimientos quirúrgicos $\mathrm{y}$, además, el número de pacientes con diagnóstico de cirrosis está en aumento en los últimos años.

Clásicamente, la evaluación del riesgo quirúrgico se ha realizado con el puntaje del Child-Turcott-Pugh (CTP) y se ha descrito que el riesgo promedio de muerte de un paciente cirrótico que se somete a una cirugía abdominal es del $11 \%$ al $28 \%$, en comparación con $1 \%$ de los pacientes no cirróticos $(2,3)$. Sin embargo, cuando se discrimina por el CTP, se reporta que la mortalidad a 30 días puede ser incluso hasta del $80 \%(4,5)$, con unos porcentajes que permanecen altos a pesar de la mejoría en los últimos años. Adicionalmente, la mayoría de los estudios con respecto a este tópico tiene limitaciones: son retrospectivos, con pocos pacientes y la mayoría de los pacientes son cirróticos CTP A, con lo cual los resultados y la aplicación de la evidencia debe tomarse con precaución.

El objetivo de este artículo es revisar las estrategias de evaluación del riesgo quirúrgico y manejo de pacientes cirróticos que serán sometidos a procedimientos quirúrgicos no hepáticos.

\section{EFECTOS DE LA ANESTESIA Y LA CIRUGÍA EN EL HÍGADO}

La enfermedad hepática puede afectar significativamente el metabolismo de los anestésicos y algunos medicamentos utilizados durante la cirugía: se altera su distribución, metabolismo y excreción. Es ampliamente conocido el potencial hepatotóxico del halotano, el cual puede generar hepatitis aguda inmunoalérgica incluso con insuficiencia hepática aguda (6). En los pacientes con hepatopatía deben preferirse otros agentes anestésicos como isoflurano, desflurano y sevoflurano, que tienen menor metabolismo hepático, así como el uso de propofol por su vida media corta. También debe tenerse precaución con opiáceos y benzodiacepinas como morfina, oxicodona, diazepam y midazolam, ya que su metabolismo con la enfermedad hepática es muy lento, con el consecuente riesgo de depresión neurológica prolongada y precipitación de encefalopatía hepática; por lo que si es necesario el uso de este tipo de agentes, se recomienda remifentanilo y oxacepam. En cuanto a los relajantes musculares se prefiere el uso de atracurio y cisatracurio porque su eliminación no depende del hígado ni del riñón. Cuando sea posible debe preferirse la anestesia regional y después de cirugía son frecuentes los cambios leves en las pruebas bioquímicas hepáticas, los cuales no tienen ningún impacto en la función hepática residual (7).
Los pacientes cirróticos se caracterizan por una circulación hiperdinámica con aumento del gasto cardíaco y disminución de la resistencia vascular sistémica. Durante la cirugía, los agentes anestésicos pueden reducir el flujo sanguíneo hepático en un $30 \%$ a $50 \%$ después de la inducción; son frecuentes los episodios de hipotensión, hemorragia y el uso de fármacos vasoactivos, lo cual lleva a una disminución significativa de la perfusión hepática y hace al hígado cirrótico más susceptible a la lesión hipóxicoisquémica. Además, los pacientes con enfermedad hepática avanzada puedan estar con ascitis severa, hidrotórax hepático, hipertensión portopulmonar y síndrome hepatopulmonar; todos estos factores son relevantes para la presencia de hipoxemia y dificultad en la ventilación mecánica de estos individuos.

\section{EVALUACIÓN DEL RIESGO QUIRÚRGICO}

La evaluación del riesgo quirúrgico en pacientes con cirrosis hepática se basa en 3 factores principales: valoración de la función hepática, la urgencia del procedimiento y el tipo de procedimiento. Los pacientes cirróticos tienen alto riesgo quirúrgico no solamente por la cirrosis en si misma sino por la presencia de coagulopatía, desnutrición, disfunción inmune, cardiomiopatía del cirrótico, alteraciones pulmonares y renales. Adicionalmente, en el estudio americano más importante con respecto al riesgo quirúrgico en procedimientos electivos se pudo determinar que la presencia de hipertensión portal clínicamente significativa (varices esofágicas, ascitis, encefalopatía) es el factor primordial relacionado con mortalidad, morbilidad, estancia hospitalaria y costos (8).

Se considera que existe contraindicación para cirugía si el paciente se encuentra con hepatitis aguda, especialmente si es severa (índice internacional normalizado [INR] $>1,5$ ); hepatitis alcohólica; o insuficiencia hepática aguda, en la que se ha descrito mortalidad del $10 \%$ al $50 \%$. En cuanto a la urgencia del procedimiento, se ha determinado que cuando es necesaria una cirugía mayor de emergencia (ASA V), la mortalidad es del $50 \%$ al $100 \%$, con grandes diferencias cuando se compara con los procedimientos electivos, por lo que debe discutirse con el paciente, la familia y el equipo quirúrgico si se asume el riesgo de la intervención urgente teniendo en cuenta estos pobres resultados. Los pacientes cirróticos CTP C tienen contraindicación para todo procedimiento quirúrgico mayor.

La evaluación prequirúrgica debe incluir la clasificación de la Sociedad Americana de Anestesiología (ASA), los cirróticos compensados se clasifican en ASA III y los descompensados ASA IV, por lo cual desde el inicio el riesgo quirúrgico es considerable. En un estudio de la clínica Mayo se encontró que la clasificación ASA era el mejor 
predictor de la mortalidad postoperatoria a los 7 días, en especial cuando se combina con la edad ( $>70$ años) y el MELD (9). Precisamente con los resultados de ese estudio se creó una herramienta en línea para ayudar a los clínicos a determinar de una forma un poco más precisa el riesgo de mortalidad postoperatoria en cirugía mayor (abdominal, cardíaca, vascular y ortopédica) al cual se puede acceder a través del siguiente enlace: www.mayoclinic.org/medicalprofessionals/model-end-stage-liver-disease/post-operative-mortality-risk-patients-cirrhosis.

Los puntajes CTP y MELD son modelos pronósticos usados para evaluar la severidad de la enfermedad hepática y tratar de estratificar el riesgo. En estudios hasta de la década de los noventa, las tasas de mortalidad postoperatoria fueron del $10 \%, 30 \%$ y $76 \%$ a $82 \%$ en cirróticos CTP A, B y C, respectivamente $(4,5)$; sin embargo, la precisión del puntaje CTP no es la mejor por la inclusión de variables subjetivas como ascitis y encefalopatía hepática. En los últimos años se han reportado mejores resultados como en un estudio reciente en el que se encontró una mortalidad del $2 \%$ en CTP A y del $12 \%$ en CTP B-C en cirugías abdominales como colecistectomía, herniorrafias y colectomías; pero en pacientes bien seleccionados y en procedimientos realizados por cirujanos entrenados en intervenciones en pacientes con hipertensión portal (10). De acuerdo con estos resultados, los pacientes con CTP A no tienen problema en cirugías electivas, los CTP $\mathrm{C}$ tienen contraindicación para procedimientos quirúrgicos y los CTP B podrían ser llevados a cirugía, pero requieren una rigurosa evaluación prequirúrgica y mejores herramientas para tomar la decisión.

El puntaje MELD es un modelo estadístico creado inicialmente para evaluar la mortalidad postderivación portosistémica intrahepática transyugular (TIPS) y ulteriormente aplicado para la priorización de pacientes en lista para trasplante hepático (11), y en los últimos años se ha convertido en una herramienta fundamental en la estratificación de pacientes con enfermedades hepáticas en diferentes escenarios. En cuanto a la evaluación prequirúrgica, se ha comparado con el CTP en varios estudios y se ha encontrado que tiene mejor rendimiento para predecir desenlaces no favorables con riesgo significativo que empieza con MELD >10; sin embargo, los puntos de corte de MELD $>14$ o 15 son más específicos $(10,12)$. En un estudio reciente se encontró que los pacientes con MELD $>15$ y albúmina $<2,5 \mathrm{~g} / \mathrm{L}$ tenían una mortalidad de $60 \%$ frente a $14 \%$, con una precisión superior al puntaje CTP (10). Adicionalmente, el MELD se ha asociado como el mejor predictor de la mortalidad posquirúrgica a 30 y 90 días (Tabla 1) (9). De acuerdo con estos estudios, se considera que los pacientes con MELD $<10$ pueden ser sometidos a procedimientos quirúrgicos sin mayores problemas, aquellos con MELD >15 y albúmina <2,5 g/L tienen contrain- dicación para cirugía y los pacientes con MELD de 10 a 15 pueden ser llevados a cirugía con una evaluación estricta y en centros especializados.

Tabla 1. Relación entre el puntaje MELD y la mortalidad postoperatoria

\begin{tabular}{lccc}
\hline \multirow{2}{*}{ Puntaje MELD } & \multicolumn{3}{c}{ Mortalidad (\%) } \\
\cline { 2 - 4 } & $\mathbf{7}$ días & $\mathbf{3 0}$ días & $\mathbf{9 0}$ días \\
\hline $0-7$ & 1,9 & 5,7 & 9,7 \\
$8-11$ & 3,3 & 10,3 & 17,7 \\
$12-15$ & 7,7 & 25,4 & 32,3 \\
$15-20$ & 14,3 & 44 & 55 \\
$20-25$ & 23 & 53 & 66 \\
$>26$ & 30 & 90 & 90 \\
\hline
\end{tabular}

Tomado de: Teh SH et al. Gastroenterology. 2007;132(4):1261-9.

Las comorbilidades son importantes, especialmente la insuficiencia renal, enfermedad cardiovascular y diabetes mellitus.

\section{TIPO DE PROCEDIMIENTO}

En los pacientes cirróticos que necesitan una cirugía deben preferirse los procedimientos mínimamente invasivos. Aunque en el pasado se consideró que la laparoscopia estaba contraindicada en pacientes cirróticos este concepto fue reevaluado y siempre que sea posible debe considerarse este abordaje. Algunos autores han realizado modificaciones en la técnica para evitar complicaciones como por ejemplo en la localización de los trocar para evitar la perforación inadvertida de las varices de la pared abdominal. Existe suficiente experiencia y estudios en colecistectomía laparoscópica (13, 14), herniorrafias, esplenectomías, colectomías, derivación gástrica y apendicetomías, entre otras (15). De todas maneras debe reconocerse que los procedimientos laparoscópicos en cirróticos tienen mayor posibilidad de convertirse a una cirugía abierta, requerir mayor tiempo quirúrgico y tener mayor sangrado cuando se comparan con los no cirróticos.

\section{CIRUGÍAS ABDOMINALES}

Las cirugías abdominales en los pacientes cirróticos son las más frecuentes y, por consiguiente, es donde se encuentra más información. Ya se comentó que se prefieren los procedimientos laparoscópicos y el uso de instrumentos que faciliten la coagulación como el escalpelo harmónico. Además de asociarse con disminución de la mortalidad, los procedimientos mínimamente invasivos se han relacionado con disminución de morbilidad: infecciones, dehiscencia de heridas, sangrados, menor tiempo quirúrgico y menor estancia hospitalaria.

Los pacientes cirróticos tienen mayor incidencia de colelitiasis que la población general; afortunadamente, la mayoría 
de ellos permanece asintomática y no necesita manejo quirúrgico. En pacientes cirróticos CTP A o B sometidos a colecistectomía laparoscópica, la morbilidad es del $5 \%$ al $10 \%$ yla mortalidad es $<2 \%$, la coexistencia de enfermedad cardiovascular es el principal factor de riesgo asociado con mortalidad (16). Por el contrario, los cirróticos CTP C tienen una mortalidad prohibitiva, por lo cual en el caso de colecistitis aguda se recomienda el manejo antibiótico; y en casos de piocolecisto, puede optarse por colecistostomía percutánea (17).

Las hernias de pared abdominal son 4 veces más frecuentes en los pacientes cirróticos que en la población general, especialmente si los pacientes tienen ascitis. En un estudio de más de 900 pacientes cirróticos CPT A o B la mortalidad global fue de 5,7\% para el manejo quirúrgico de hernia inguinal, con una morbilidad aceptable y una tasa de recurrencia del 8 $\%$ (18). Los resultados en hernias umbilicales son similares, pero en el caso de procedimientos urgentes la mortalidad se aumentó 5 veces frente al procedimiento electivo. En reportes recientes, la mortalidad fue de $0 \%$ en centros de referencia con gran experiencia y en pacientes seleccionados. Las herniorrafias umbilicales frecuentemente requieren el uso de mallas, pero debe tenerse en cuenta que en algunos estudios se asociaron con mayor tasa de infecciones.

En cuanto a la cirugía colorrectal, se ha reportado una mortalidad del $13 \%$ al $35 \%$ con una morbilidad del 50 $\%$, siendo los factores relacionados con mal pronóstico los pacientes cirróticos CTP B o C y aquellos llevados a cirugía urgente por cuadros de obstrucción intestinal, sangrado o perforación (19). En los pacientes urgentes con obstrucción intestinal podría optarse por stent colónico, y en el caso de sangrado por úlcera o tumor, se debe pensar en el manejo endoscópico. En el caso de gastrectomías la mortalidad es del $14 \%$ a $29 \%$, con una morbilidad del $53 \%$ para cirróticos CTP A y del 68 \% para CTP B (8).

\section{CIRUGÍAS CARDÍACAS Y VASCULARES}

La mortalidad de cirugía cardíaca electiva en pacientes cirróticos CTP A es $0 \%-11 \%$, CTP B es $18 \%-67 \%$ y CTP C es $67 \%-100 \%$; y la morbilidad es del $25 \%-50 \%, 50$ $\%-100 \%$ y del $100 \%$ en CTP A, B y C, respectivamente (20). De acuerdo con lo anterior, los pacientes cirróticos CTP A podrían ser llevados a cirugías electivas en casos bien seleccionados incluso con el uso de derivación cardiopulmonar, pero los pacientes CTP B y $\mathrm{C}$ tienen un riesgo quirúrgico prohibitivo. Otros predictores de mortalidad son la edad $>60$ años, sexo femenino, ascitis e insuficiencia cardíaca congestiva; con una mortalidad $>50 \%$ para pacientes con 2 o más de los anteriores factores.

La reparación de aneurismas de la aorta abdominal es posible en cirróticos CTP A, aunque estos pacientes tienen mayor necesidad de transfusiones, mayor tiempo quirúrgico, mayor estancia hospitalaria y costos cuando se compara con los no cirróticos (21). Los pacientes cirróticos con CTP B o MELD > 10 tuvieron asociación con morbimortalidad.

\section{CIRUGÍAS ORTOPÉDICAS Y TRAUMA}

Los pacientes cirróticos sometidos a cirugías ortopédicas mayores electivas en cadera o rodilla tienen una morbilidad $>25 \%$ con una mortalidad del $4 \%, 14 \%$ y $100 \%$ para cirróticos CTP A, B y C, respectivamente; sin embargo, los resultados son significativamente mejores que cuando se realizan estos procedimientos de urgencia cuya morbilidad es superior al $80 \%$ y la mortalidad puede llegar al $60 \%$ (22).

Los pacientes cirróticos con traumas tienen el doble de mortalidad que los pacientes no cirróticos. Adicionalmente, los pacientes con trauma cerrado de abdomen que requieren laparotomía de urgencia y MELD > 17 tienen una mortalidad postoperatoria del $85 \%$ (23).

\section{CUIDADOS PERIOPERATORIOS}

Los pacientes cirróticos son un grupo especial de pacientes que requieren cuidados pre, intra y postoperatorios para lograr resultados favorables con los procedimientos quirúrgicos. La evaluación prequirúrgica es fundamental para identificar si el paciente ha tenido complicaciones en el pasado, el tratamiento recibido y si actualmente está con manifestaciones de descompensación.

En todo paciente cirrótico que sea llevado a cirugía debe optimizarse el manejo y controlar de forma efectiva sus complicaciones (Tabla 2).

Es habitual que los pacientes cirróticos tengan alteraciones de las pruebas tradicionales de coagulación, pero en la actualidad está totalmente demostrado que en estos pacientes la balanza de la coagulación esta rebalanceada (lo que explica que incluso tienen eventos trombóticos) y, por tanto, este tipo de alteraciones no debe ser una contraindicación para cirugía. Lo ideal para evaluar la función plaquetaria y la coagulación en los cirróticos es el uso de pruebas dinámicas y globales como la tromboelastografía (TEG). En un estudio reciente se evaluó la TEG para definir una estrategia de transfusión guiada por TEG para procedimientos invasivos y se logró determinar que el uso de TEG lograba reducir la necesidad de transfusiones en $90 \%$ sin un incremento en las complicaciones de sangrado (24), pero deben esperarse nuevos estudios para validar esta información.

La trombocitopenia es casi universal en los pacientes con cirrosis hepática, aunque la transfusión prequirúrgica está indicada cuando el recuento de plaquetas es $<50000$ plaquetas $/ \mu \mathrm{L}$ o podría considerarse en cirugías con alto riesgo de sangrado con recuentos entre 50 000-80 000 plaquetas/ $\mu \mathrm{L}$. En cirróticos con insuficiencia renal podría optarse 
Tabla 2. Recomendaciones de manejo perioperatorio del paciente cirrótico

\begin{tabular}{ll}
\hline \multicolumn{1}{c}{ Complicación } & \multicolumn{1}{c}{ Intervención } \\
\hline Ascitis & Realizar paracentesis para descartar peritonitis bacteriana espontánea. \\
& Si hay ascitis moderada a severa, realizar paracentesis de grandes volúmenes y reposición con albúmina. \\
& Iniciar/ajustar diuréticos. \\
& Hacer dieta hiposódica. \\
& Iniciar lactulosa para garantizar 2 a 4 deposiciones al día. \\
& Si persiste la encefalopatía, adicionar rifaximina. \\
& Evitar el uso de opiáceos y benzodiacepinas. \\
& Iniciar/ajustar bloqueante $\beta$ \\
Várices esofágicas & Hacer ligadura endoscópica prequirúrgica en várices de alto riesgo o con antecedentes de sangrado. \\
& Reponer la vitamina $\mathrm{K}: 10$ mg/día por 3 días. \\
Recomendaciones generales & Optimizar la nutrición, sin restricción de proteínas $(1,2-1,5 \mathrm{~g} / \mathrm{kg} / \mathrm{d})$. \\
& Evitar medicamentos hepatotóxicos o nefrotóxicos. \\
& Monitorizar y corregir alteraciones hidroelectrolíticas. \\
\hline
\end{tabular}

por la aplicación prequirúrgica de desmopresina. Otra opción es el uso de eltrombopag, el cual se relaciona con el incremento del recuento de plaquetas y disminución de la necesidad de transfusiones, pero se asoció con eventos trombóticos portales, por lo cual no se recomienda su uso (25). No hay estudios apropiados para evaluar los resultados de transfusión de plasma fresco congelado en cirróticos que van a ser llevados a cirugía, pero en términos generales se transfunde cuando el INR es $>1,5$. Una estrategia económica y segura es la aplicación de vitamina $\mathrm{K} 10 \mathrm{mg}$ /día por 3 días para corregir el déficit de vitamina $\mathrm{K}$, que no es raro en los cirróticos. Aunque no hay una meta específica de hemoglobina en los cirróticos que serán llevados a cirugía, se recomienda una estrategia restrictiva para evitar el exceso de transfusiones porque esta se relaciona con el incremento de la presión portal y mayor sangrado.

Los TIPS pueden controlar la hipertensión portal y, de esta forma, mejorar los resultados posquirúrgicos de los cirróticos. La principal indicación es la presencia de ascitis o várices esofágicas en pacientes que requieren cirugías abdominales y tienen un MELD $<15$. Los estudios han demostrado que los pacientes pudieron ser llevados a cirugías mayores como resecciones oncológicas con intención curativa y tuvieron menos sangrado, transfusiones y ascitis postoperatoria, y se reportó una supervivencia aceptable en el seguimiento a mediano plazo (26).

En el cuidado postoperatorio debe evitarse tanto el exceso como el déficit de líquidos endovenosos ya que estos se relacionan con edemas, ascitis o insuficiencia renal, respectivamente. En pacientes cirróticos con ascitis y cirugía abdominal, la recurrencia de ascitis es casi universal, no son raras las fístulas de líquido ascítico y hay mayor riesgo de dehiscencia de la herida quirúrgica; por tanto, se reco- mienda el uso temprano de diuréticos, realizar paracentesis a necesidad y evitar el uso prolongado de drenajes abdominales para disminuir el riesgo de infecciones, hipovolemia y desnutrición. En estos casos, el uso de albúmina es de gran utilidad gracias a sus propiedades no solamente oncóticas sino también inmunorreguladoras, antioxidantes y de transporte de sustancias y medicamentos. Debe evitarse el uso de opiáceos y benzodiacepinas para reducir el riesgo de encefalopatía y, con el uso de lactulosa, garantizar el número adecuado de deposiciones; y en los pacientes intolerantes a lactulosa puede usarse polietilenglicol. Las infecciones en el cirrótico son frecuentes después de cirugía así que es necesario tener un bajo umbral para la búsqueda y diagnóstico de las mismas, siendo los focos más frecuentes la cavidad abdominal, infecciones urinarias, neumonía y bacteriemias espontáneas.

Los pacientes cirróticos frecuentemente están desnutridos y requieren soporte nutricional previo y posterior a la cirugía, con inicio de la vía oral lo antes posible. Se recomienda una dieta hiposódica, con un requerimiento calórico de $30-35 \mathrm{kcal} / \mathrm{kg} /$ día y una ingesta de proteínas de 1,2-1,5 g/ $\mathrm{kg} /$ día. Adicionalmente, debe promoverse la movilización temprana, terapia de incentivo respiratorio y el inicio temprano de profilaxis contra la trombosis venosa profunda.

\section{CONCLUSIONES}

Los pacientes con cirrosis hepática y especialmente aquellos con hipertensión portal tienen un riesgo quirúrgico superior a la población general. Cuando un paciente cirrótico requiere una cirugía es necesario realizar una evaluación exhaustiva y minuciosa por parte del clínico para determinar la función hepática y la urgencia y el tipo de procedimiento, de tal forma 
que pueda decidirse si el paciente puede ser llevado de forma segura al procedimiento y garantizar las medidas perioperatorias necesarias para incrementar el éxito de los procedimientos quirúrgicos.

\section{Fuente de apoyo financiero}

Ninguna.

\section{REFERENCIAS}

1. Friedman LS. The risk of surgery in patients with liver disease. Hepatology. 1999;29(6):1617-23. doi: 10.1002/ hep.510290639.

2. Neeff H, Mariaskin D, Spangenberg HC, Hopt UT, Makowiec F. Perioperative mortality after non-hepatic general surgery in patients with liver cirrhosis: an analysis of 138 operations in the 2000s using Child and MELD scores. J Gastrointest Surg. 2011;15(1):1-11. doi: 10.1007/s11605-010-1366-9.

3. Aranha GV, Sontag SJ, Greenlee HB. Cholecystectomy in cirrhotic patients: a formidable operation. Am J Surg. $1982 ; 143(1): 55-60$.

4. Garrison RN, Cryer HM, Howard DA, Polk HC Jr. Clarification of risk factors for abdominal operations in patients with hepatic cirrhosis. Ann Surg. 1984;199(6):648-55.

5. Mansour A, Watson W, Shayani V, Pickleman J. Abdominal operations in patients with cirrhosis: still a major surgical challenge. Surgery. 1997;122(4):730-5.

6. Walton B, Simpson BR, Strunin L, Doniach D, Perrin J, Appleyard AJ. Unexplained hepatitis following halothane. Br Med J. 1976;1(6019):1171-6.

7. O'Leary JG, Yachimski PS, Friedman LS. Surgery in the patient with liver disease. Clin Liver Dis. 2009;13(2):21131. doi: 10.1016/j.cld.2009.02.002.

8. Csikesz NG, Nguyen LN, Tseng JF, Shah SA. Nationwide volume and mortality after elective surgery in cirrhotic patients. J Am Coll Surg. 2009;208(1):96-103. doi: 10.1016/j.jamcollsurg.2008.09.006.

9. Teh SH, Nagorney DM, Stevens SR, Offord KP, Therneau TM, Plevak DJ, et al. Risk factors for mortality after surgery in patients with cirrhosis. Gastroenterology. 2007;132(4):1261-9. doi: 10.1053/j.gastro.2007.01.040.

10. Telem DA, Schiano T, Goldstone R, Han DK, Buch KE, Chin $\mathrm{EH}$, et al. Factors that predict outcome of abdominal operations in patients with advanced cirrhosis. Clin Gastroenterol Hepatol. 2010;8(5):451-7, quiz e58. doi: 10.1016/j.cgh.2009.12.015.

11. Malinchoc M, Kamath PS, Gordon FD, Peine CJ, Rank J, ter Borg PC. A model to predict poor survival in patients undergoing transjugular intrahepatic portosystemic shunts. Hepatology. 2000;31(4):864-71. doi: 10.1053/he.2000.5852.

12. Befeler AS, Palmer DE, Hoffman M, Longo W, Solomon $\mathrm{H}, \mathrm{Di}$ Bisceglie AM. The safety of intra-abdominal surgery in patients with cirrhosis: model for end-stage liver disease score is superior to Child-Turcotte-Pugh classification in predicting outcome. Arch Surg. 2005;140(7):650-4. doi: 10.1001/archsurg.140.7.650.
13. Yeh CN, Chen MF, Jan YY. Laparoscopic cholecystectomy in 226 cirrhotic patients. Experience of a single center in Taiwan. Surg Endosc. 2002;16(11):1583-7. doi: 10.1007/ s00464-002-9026-0.

14. Cucinotta E, Lazzara S, Melita G. Laparoscopic cholecystectomy in cirrhotic patients. Surg Endosc. 2003;17(12):195860. doi: $10.1007 /$ s00464-002-8852-4.

15. Cobb WS, Heniford BT, Burns JM, Carbonell AM, Matthews $\mathrm{BD}$, Kercher KW. Cirrhosis is not a contraindication to laparoscopic surgery. Surg Endosc. 2005;19(3):418-23. doi: 10.1007/s00464-004-8722-3.

16. Bloch RS, Allaben RD, Walt AJ. Cholecystectomy in patients with cirrhosis. A surgical challenge. Arch Surg. 1985;120(6):669-72.

17. Byrne MF, Suhocki P, Mitchell RM, Pappas TN, Stiffler HL, Jowell PS, et al. Percutaneous cholecystostomy in patients with acute cholecystitis: experience of 45 patients at a US referral center. J Am Coll Surg. 2003;197(2):206-11.

18. Hurst RD, Butler BN, Soybel DI, Wright HK. Management of groin hernias in patients with ascites. Ann Surg. 1992;216(6):696-700.

19. Gervaz P, Pak-art R, Nivatvongs S, Wolff BG, Larson D, Ringel S. Colorectal adenocarcinoma in cirrhotic patients. J Am Coll Surg. 2003;196(6):874-9. doi: 10.1016/S10727515(03)00117-0.

20. Modi A, Vohra HA, Barlow CW. Do patients with liver cirrhosis undergoing cardiac surgery have acceptable outcomes? Interact Cardiovasc Thorac Surg. 2010;11(5):630-4. doi: $10.1510 /$ icvts.2010.241190.

21. Marrocco-Trischitta MM, Kahlberg A, Astore D, Tshiombo G, Mascia D, Chiesa R. Outcome in cirrhotic patients after elective surgical repair of infrarenal aortic aneurysm. J Vasc Surg. 2011;53(4):906-11. doi: 10.1016/j.jvs.2010.10.095.

22. Cohen SM, Te HS, Levitsky J. Operative risk of total hip and knee arthroplasty in cirrhotic patients. J Arthroplasty. 2005;20(4):460-6. doi: 10.1016/j.arth.2004.05.004.

23. Lin BC, Fang JF, Wong YC, Hwang TL, Hsu YP. Management of cirrhotic patients with blunt abdominal trauma: analysis of risk factor of postoperative death with the Model for EndStage Liver Disease score. Injury. 2012;43(9):1457-61. doi: 10.1016/j.injury.2011.03.057.

24. De Pietri L, Bianchini M, Montalti R, De Maria N, Di Maira T, Begliomini B, et al. Thrombelastography-guided blood product use before invasive procedures in cirrhosis with severe coagulopathy: A randomized, controlled trial. Hepatology. 2016;63(2):566-73. doi: 10.1002/hep.28148.

25. Afdhal NH, Giannini EG, Tayyab G, Mohsin A, Lee JW, Andriulli A, et al. Eltrombopag before procedures in patients with cirrhosis and thrombocytopenia. $\mathrm{N}$ Engl J Med. 2012;367(8):716-24. doi: 10.1056/NEJMoa1110709.

26. Lahat E, Lim C, Bhangui P, Fuentes L, Osseis M, Moussallem $\mathrm{T}$, et al. Transjugular intrahepatic portosystemic shunt as a bridge to non-hepatic surgery in cirrhotic patients with severe portal hypertension: a systematic review. HPB (Oxford). 2018;20(2):101-109. doi: 10.1016/j.hpb.2017.09.006. 